Research Article

\title{
Rail Optimization of Noncircular Curve of Crane Turning Based on Quasiquartic Bezier Curve with Three Shape Parameters
}

\author{
Gang Liang, ${ }^{1}$ Zhangjie $W u\left(D,{ }^{1}\right.$ and Weikun $Y u^{2}$ \\ ${ }^{1}$ Logistics Engineering College, Shanghai Maritime University, Shanghai 201306, China \\ ${ }^{2}$ Shanghai Zhenhua Heavy Industry Co., Ltd, Shanghai 200125, China \\ Correspondence should be addressed to Zhangjie Wu; wzj19950423@163.com
}

Received 9 December 2019; Revised 31 August 2020; Accepted 16 December 2020; Published 29 December 2020

Academic Editor: Luigi Rodino

Copyright (c) 2020 Gang Liang et al. This is an open access article distributed under the Creative Commons Attribution License, which permits unrestricted use, distribution, and reproduction in any medium, provided the original work is properly cited.

In order to solve the problems of rail gnawing and jamming during turning of rail crane, a noncircular curve scheme of the crane based on Bezier curve is proposed. In the scheme, the quasiquartic Bezier curve with three shape parameters is chosen as the turning curve of the inner rail. According to the single-wheel and multiwheel situation of the crane, the tracks of the front and rear points on the outer side are calculated through the geometric relationship of the traveling mechanism of the crane cart. Taking the minimum deviation of the front and back points as the objective function of optimization, the optimal parameters of Bezier curve are searched by the multistart point heuristic global optimization algorithm, and the outer rail trajectory is fitted by Hermite interpolation. The calculation results show that the maximum deviation of the front and rear points on the outside of the crane during the turning process decreases significantly when the quartic Bezier curve is used as the turning track compared with the traditional circular turning track. When the quasiquartic Bezier curve with three shape parameters is used as the inner rail, the deviation can be further reduced by adjusting the three parameters. In addition, it is also analyzed the specific influence of turning parameters on the deviation. Finally, ADAMS is used to carry out dynamic simulation experiment and demonstrate the calculation.

\section{Introduction}

Multiple berths tend to cover modern large-scale ports, and the distribution of berths largely depends on the natural conditions on the shores of the ports. In order to realize the operation of a quayside container crane for multiple berths, the quayside container cranes need to be able to move between multiple berths. Usually, the crane only needs to run on the straight track, but some ports are not unidirectional to the sea, and berths cannot be linearly distributed, so cranes need to turn and move. In addition, various types of rail-operated crane such as bridge crane, gantry crane, tower crane, and gantry crane operate along straight line track in general engineering practice. However, in some special cases, such as the actual need for crane operation or due to the limitations of the natural conditions of the installation site, cranes are sometimes required to run on curved tracks, such as gantry cranes used in the construction of hydropower stations, tower cranes used in construction sites, and so on, which may require cranes to run on curved tracks. However, during the turning process of the railoperated crane, there will be a deviation in the trajectory of the front and rear wheels, especially the multiwheel operating system. If the deviation is small enough, it can be offset by the gap between the rim and the track, but if the deviation is too large, the gap is not enough to offset this deviation, which will cause dangerous situations such as gnawing and jamming between the wheel edge and the track, so the turning problem of the crane has increasingly become the focus of the industry with the development of the crane industry.

At present, the passing problem of the turning track of the crane cart is mainly through the adjusting the crane parameters such as the frame size and the wheel tread width or adding the eccentric bearing to achieve a smooth cornering. Although these methods are effective, due to fewer 
adjustable parameters and smaller adjustable range, the result is that the turning radius of the rail will be too large or the related motion control mechanism will be increased, which may cause crane running stability problems, thereby increasing the difficulty of design and manufacturing costs.

The design studies of traditional crane turning tracks are based on concentric circular or simply modified on concentric circular tracks. Gu [1] studied the turning track and cornering conditions of the gantry crane operating mechanism of the hydropower station for the first time, deduced the critical radius equation of the single-wheel and twowheel pure arc-turning, and gave a set of rail correction methods. $\mathrm{Ni}$ [2] discussed and analyzed the basic problems of turning track design of loading and unloading bridges and proposed a turning design scheme that combines the modified running track with the widened wheel tread. Xu [3] combined with the field situation to check the minimum radius of curvature from the application point of view and discussed the fulcrum structure types of four different cart operating mechanisms, respectively. The results show that the crane with upper vertical fulcrum structure is more suitable for concentric circular track. Zhang et al. [4] proposed the verification method for the crane running on the curve. Compared with the design method proposed by $\mathrm{Xu}$, this method takes the contact between the wheel rim and the track into account more carefully. Wang [5] targeted several different types of turning carts currently designed and manufactured by Shanghai Zhenhua Port Machinery, mainly considering the calculation of the minimum turning radius and the maximum deviation, and introduced several commonly used methods such as changing base distance, using vertical axle rotary carts, and increasing eccentric bearings. At the same time, he pointed out that the modified track scheme which has emerged in recent years has two significant advantages. First, it is suitable for the small docks; second, it does not use eccentric bearings, avoiding additional bending moments during bending, and the track tread at the corners can also be appropriately reduced.

The function value and the first derivative of the concentric circular turning track at the junction where the straight line meets the arc are continuous, but the deviation at the junction still increases sharply, which is due to the discontinuity of the second derivative of both, so the continuity of the first derivative in mathematics cannot meet the requirement of smooth transition of crane. The curve of the turning rail must have the continuity of the second derivative to meet this requirement. The so-called second derivative continuity is that the curvature radius of a curve is continuous, the curvature radius of a straight line is infinite, and the curvature radius of the arc is a certain value, and the two are obviously discontinuous. For this purpose, a transition curve [6-8] is introduced between the two to satisfy the continuous change of the curvature radius.

In recent years, a new type of crane turning scheme has emerged in the crane manufacturing industry, which is called modified orbital scheme. The focus of this method is on the design of its curve. The curve of the modified track is not composed of a simple arc, but a nonarc curve [9]. Chen et al. [10] designed a complete noncircular turning rail by using clothoid as the transition curve and deduced the complete orbital equation of the clothoid. Based on this, the multiwheel correction principle of the noncircular rail was studied.

The Bezier curve is one of the spline curves, which has very good geometric continuity and controllability. In the research of trajectory planning based on Bezier curve, Huang [11] applied rational Bezier curve to robot trajectory planning and designed and implemented an algorithm based on rational Bezier curve. Chen et al. [12] proposed a trajectory planning method based on fourth-order Bezier curve to generate a local feasible trajectory that satisfies the initial and objective state constraints of the unmanned vehicle. In this method, the trajectory planning problem is first decomposed into two subproblems: rail planning and velocity planning. Han et al. [13] proposed a path planning for the anticollision behavior of electronic vehicles based on Bezier curve. Bae et al. [14] introduced a path planning and tracking method for autonomous vehicles based on fifth-order Bezier curves and used Matlab to generate the single and double lanes.

Bezier curve can smoothly transit with the straight line segment, and its continuity can be changed by adjusting the parameters of control points. In addition, in recent years, by introducing various Bernstein basis functions with parameters, the Bezier curve can achieve the effect of local adjustment without changing the position of the control points. Han and Liu [15] introduced shape parameters into the basic function to extend the quartic Bezier curve. By changing the value of parameter in the numerical range, the degree of the curve close to the control polygon can be adjusted. In the extension of basis function of cubic Bezier curve, Hang et al. [16] introduced two parameters to make the curve more flexible in shape adjustability. Similarly, Wu et al. [17-20] also carried out corresponding research on the extension of the quartic Bezier curve, respectively, introducing single, double, and three parameters into the basis function. The new curve constructed not only has similar characteristics with the quartic Bezier curve but also has more flexibility and convenience in adjusting the shape.

According to the characteristics of the noncircular curve of crane turning, the quasiquartic Bezier curve with three shape parameters is introduced into the design of crane turning. Taking the deviation of the front and rear points of the outer rail as the objective function, the global optimization algorithm [21, 22] is adopted to optimize the parameters of the multiple control points to form the inner rail of the Bezier curve, and then the coordinate parameters of the front and rear points of the outer rail are deduced directly from the wheel coordinate parameter equation of the inner rail and the relevant parameters of the crane through the correlation of the rigid body motion. The coordinate points of the modified outer rail curve are determined according to the least squares method. In order to ensure the smoothness of the curve obtained after calculation, it is necessary to smooth the discrete points and form the optimal outer rail by the Hermite interpolation method. In this way, the optimization calculation and comparative analysis of the noncircular curved track of the single-wheel and multiwheel situation of the crane are carried out, which provides a reference for the research on the design of the noncircular curved track of the crane. 


\section{Principles of Rail Optimization}

The existing crane carts generally adopt a four-point support structure, and the wheels are mostly double-rimmed cylindrical wheels. Figure 1 shows a simplified model of the traveling mechanism of the eight-wheeled cart, where $B$ is the base distance of the crane and $S$ is the gauge distance. Figure 2 shows the balanced beam span of the eight-wheeled cart, where $K_{0}$ is the wheelbase and $K_{1}, K_{2}$, and $K_{3}$ are the spans of the first-, second-, and third-level balanced beams.

For different designs of cranes, the basic model is similar to Figure 1, except that the number of balanced beam steps and wheels on each fulcrum is different. Several different crane running mechanism models are shown in Figure 3.

There are two main ways to optimize the crane track: (1) first fix the inside trajectory and then fix the outside trajectory-take the minimum deviation of the front and rear points of the outer rail as the optimization target, so as to determine the inner rail, and then determine the outer rail from the specified inner rail; (2) first fix the outside trajectory and then fix the inside trajectory-take the minimum deviation of the front and rear points of the inner rail as the optimization target, so as to determine the outer rail, and then the inner rail is determined by the specified outer rail. The optimization scheme for the turning track of the crane is the former. The inner rail adopts the Bezier curve to obtain the coordinate matrix of the front and rear points $a$ and $b$ of the inner rail. Then, the coordinate matrix of front and rear points $c$ and $d$ of the outer rail is obtained by using the geometric structure relationship of the traveling cart. Then, the local coordinate system is established with the point a as the origin, $\mathrm{ab}$ as the horizontal axis, and ad as the vertical axis. The minimum deviation of the front and rear points of the outer rail is the optimization objective to determine the inner rail. Finally, the inner rail based on Bezier curve is determined by the minimum deviation. According to the least squares method, the coordinate points of the modified outer rail curve are calculated. In order to ensure the smoothness of the calculated curve, the discrete points are smoothed and the optimal outer rail is formed by Hermite interpolation. The ideal curve of the outer rail needs to satisfy the requirement of minimizing the deviation of the front and rear points. Since the value of the deviation is positive or negative, the central curve of the trajectory of the front and rear points is the ideal rail curve, which can meet the requirement of minimizing the deviation. It should be explained that for the single-wheel case, the front and rear points of the outer rail refer to the coordinate trajectories of the front and rear wheels on the outside of the crane; for the multiwheel case, the front and rear points of the outer rail refer to the coordinate trajectory of the front and rear rotating axles of the lower balanced beam on the outside of the crane (as shown in Figure 1 ( $c$ and $d$ )).

\subsection{Quasiquartic Bezier Curve with Three Shape Parameters}

Definition 1. $\forall t \in[0,1]$; polynomial about $t$ is named as a basis function with parameters $\lambda, \alpha$, and $\beta$, and $\lambda \in[-4,1], \beta \in[-4,6], \alpha \in[-4,1]$.

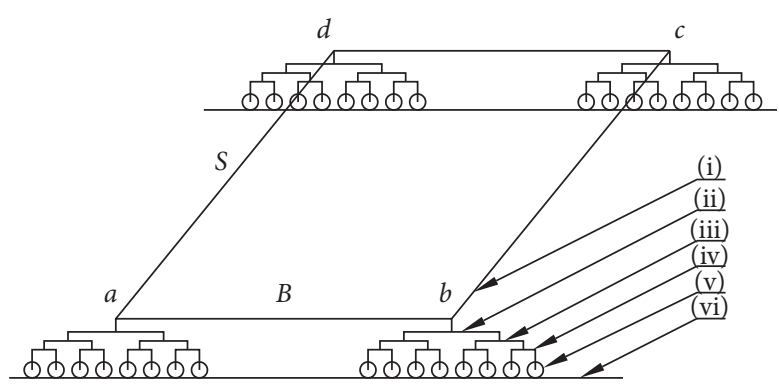

$\begin{array}{ll}\text { i. Simplified bottom } & \text { iv. First-level } \\ \text { frame of crane } & \text { balanced beam } \\ \text { ii. Third-level } & \text { v. Wheel } \\ \text { balanced beam } & \text { vi. Rail } \\ \text { iii. Second-level } & \\ \text { balanced beam } & \end{array}$

FIGURE 1: Simplified model of eight-wheeled cart: (i) simplified bottom frame of crane; (ii) third-level balanced beam; (iii) secondlevel balanced beam; (iv) first-level balanced beam; (v) wheel; (vi) rail.

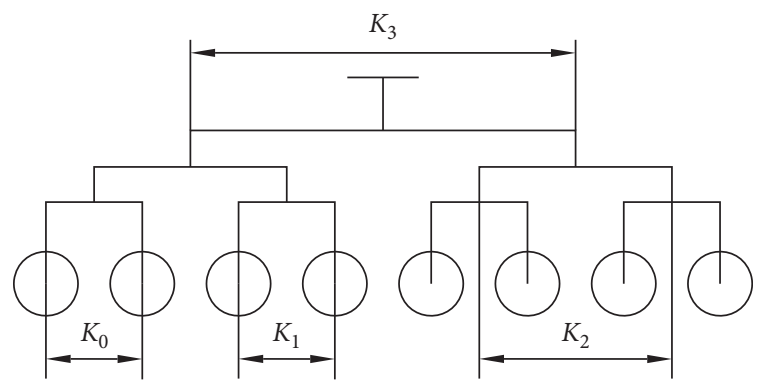

FIgURE 2: Balanced beam span of the eight-wheeled cart.

$$
\left\{\begin{array}{l}
b_{0,4}(t)=(1-\lambda t)(1-t)^{4}, \\
b_{1,4}(t)=(4+\lambda-\lambda t)(1-t)^{3} t, \\
b_{2,4}(t)=(6-\beta t)(1-t)^{2} t^{2}, \\
b_{3,4}(t)=[4+\alpha t+\beta(1-t)](1-t) t^{3}, \\
b_{4,4}(t)=(1-\alpha+\alpha t) t^{4} .
\end{array}\right.
$$

It is not difficult to see that when $\lambda=\alpha=\beta=0$, equation (1) degenerates into quartic Bernstein basis function. When $\lambda=\alpha, \beta=0$, equation (1) degenerates into a basis function with a single parameter. When $\beta=0$, equation (1) degenerates into a basis function with two parameters.

Some properties of the equation (1) are as follows:

(1) Non-negativity: $b_{j, 4} \geq 0(j=0,1,2,3,4)$.

(2) Normativity: $\sum_{j=0}^{4} b_{j, 4}(t) \equiv 1$.

(3) Properties of endpoints: $b_{j, 4}(0)=\left\{\begin{array}{ll}1, & \text { if } j=0, \\ 0, & \text { if } j \neq 0,\end{array}\right.$, $b_{j, 4}(1)= \begin{cases}1, & \text { if } j=4 \\ 0, & \text { if } j \neq 4\end{cases}$

(4) Symmetry: when $\lambda=\alpha$ and $\beta=0, b_{j, 4}(t)=b_{4-j, 4}(1-$ t) $(j=0,1,2,3,4)$. 


\begin{tabular}{|l|l|c|c|}
\hline Single-wheel & No balanced beam \\
Four-wheel \\
(two wheels are collinear)
\end{tabular}

FIgURE 3: Different crane running mechanism models.

Constructing a polynomial curve through the five points of control which are given as $P_{j} \in R^{d}(d=$ $2,3 ; j=0,1,2,3,4)$ in $t \in[0,1]$ :

$$
B(t)=\sum_{j=0}^{4} P_{j} b_{j, 4}(t)
$$

Equation (2) is named as quasiquartic Bezier curve with three shape parameters named as $\lambda, \alpha$, and $\beta$.

The quasiquartic Bezier curve determined by the five control points $P_{0}(-6,0), P_{1}(-4,4), P_{2}(0,6), P_{3}(4,2)$, and $P_{4}(6,4)$ is affected by three shape parameters as shown in Figure 4. Fixing $\alpha=\beta=0$, the virtual dotted lines from bottom to top are the corresponding patterns when $\lambda$ is taken in the order of $-4,-3,-2,-1,0,1$; fixed $\lambda=\beta=0$, the dotted lines from bottom to top are the corresponding patterns when $\alpha$ is taken in the order of $1,0,-1,-2,-3,-4$; fixing $\lambda=\alpha=0$, the solid lines from bottom to top are the corresponding patterns when $\beta$ is taken in the order of $6,4,2,0,-2,-4$. It can be seen from the figure that the three parameters have different degrees of adjustment for the shape of different parts of the curve, which provides an additional method for local adjustment of the shape of the curve.

2.2. Method of Inner Rail Forming. As shown in Figure 5, the quasiquartic Bezier curve has five control points and three parameters. In order to ensure the tangency between the curved track and the straight track, the initial and final fixed control points $p_{0}$ and $p_{4}$ are set at the junction of the two straight tracks and the curved track. The two straight tracks intersect at point $A(z, 0)$, and the angle between the straight track II and the horizontal axis is $a$. The variable control points $p_{1}$ and $p_{3}$ are located on the extension line of the two straight tracks, and $p_{2}$ is located in the triangular area

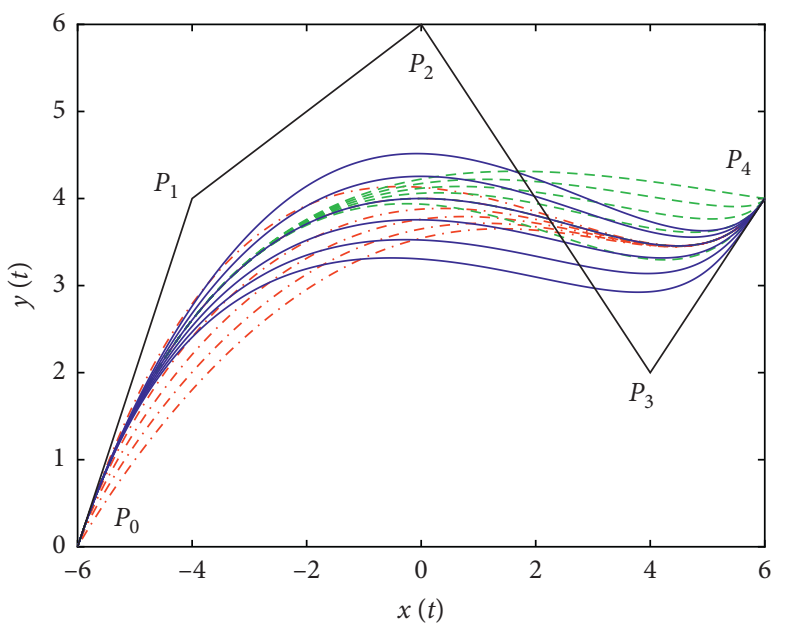

Figure 4: Effect of three parameters on the shape of the curve.

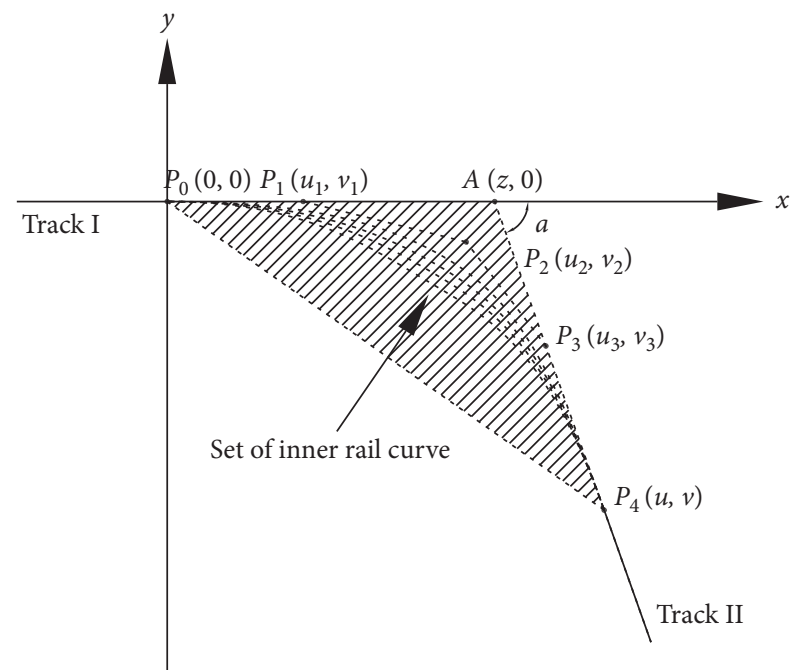

FIgURE 5: Inner rail based on quasiquartic Bezier curve. 
surrounded by the two straight tracks. By adjusting the positions of the control points $p_{1}$ and $p_{3}$ on the line segments $\mathrm{AP}_{0}$ and $\mathrm{AP}_{4}$ and the position of the $p_{2}$ in the triangular area, the set of inner rail curve can be obtained. The deviation of the front and rear points of the outer rail corresponding to each curve can be obtained according to the theoretical calculation. On this basis, the shape of the curve can be adjusted locally and corrected continuously by adjusting the values of $\lambda, \alpha$, and $\beta$. Finally, the curve corresponding to the minimum deviation is selected as the inner rail during the turning process of the crane.

Substitute the horizontal and vertical coordinate values of $P_{0}(0,0), P_{1}\left(u_{1}, v_{1}\right), P_{2}\left(u_{2}, v_{2}\right), P_{3}\left(u_{3}, v_{3}\right)$, and $P_{4}\left(u_{4}, v_{4}\right)$ into equation (2):

$$
\left\{\begin{array}{l}
x=(4+\lambda-\lambda t)(1-t)^{3} t u_{1}+(6-\beta t)(1-t)^{2} t^{2} u_{2}+[4+\alpha t+\beta(1-t)](1-t) t^{3} u_{3}+(1-\alpha+\alpha t) t^{4} u \\
y=(6-\beta t)(1-t)^{2} t^{2} v_{2}+[4+\alpha t+\beta(1-t)](1-t) t^{3} v_{3}+(1-\alpha+\alpha t) t^{4} v
\end{array}\right.
$$

\section{Calculation of the Deviation}

Calculating the deviation of front and rear points of the outer rail is divided into single-wheel (including two-wheel case) and multiwheel cases. The multiwheel case can be divided into four-wheel, six-wheel, eight-wheel cases, etc. Since the multiwheel calculating methods of the deviation are similar, we take the four-wheel case as an example to calculate and illustrate.

3.1. Single-Wheel Case. Locate the center of the wheel inside the crane (the center of the support) and calculate the coordinates of the center of the wheel on the other side, which is shown in Figure 6.

Since the constraint equations corresponding to whether the centers of the respective rotation axles enter the curve are different, it needs to be calculated and processed in segments.

(1) Wheels do not fully enter the curve: at this point, the coordinate of the inner rear wheel a of the crane is $\left(X_{a}, Y_{a}\right), X_{a} \in[-B, 0], Y_{a}=0$. The coordinate of the inner front wheel $b$ is $\left(X_{b}, Y_{b}\right)$ :

$$
\left\{\begin{array}{l}
X_{b}=\left(4+\lambda-\lambda t_{b}\right)\left(1-t_{b}\right)^{3} t_{b} u_{1}+\left(6-\beta t_{b}\right)\left(1-t_{b}\right)^{2} t_{b}^{2} u_{2}+\left[4+\alpha t_{b}+\beta\left(1-t_{b}\right)\right] \\
\left(1-t_{b}\right) t_{b}^{3} u_{3}+\left(1-\alpha+\alpha t_{b}\right) t_{b}^{4} u \\
Y_{b}=\left(6-\beta t_{b}\right)\left(1-t_{b}\right)^{2} t_{b}^{2} v_{2}+\left[4+\alpha t_{b}+\beta\left(1-t_{b}\right)\right]\left(1-t_{b}\right) t_{b}^{3} v_{3}+\left(1-\alpha+\alpha t_{b}\right) t_{b}^{4} v
\end{array}\right.
$$

(2) Wheels completely enter the curve: at this time, the coordinates of the front and rear wheels inside the crane are

$$
\left\{\begin{array}{l}
X_{a}=\left(4+\lambda-\lambda t_{a}\right)\left(1-t_{a}\right)^{3} t_{a} u_{1}+\left(6-\beta t_{a}\right)\left(1-t_{a}\right)^{2} t_{b}^{2} u_{2}+\left[4+\alpha t_{a}+\beta\left(1-t_{a}\right)\right] \\
\left(1-t_{a}\right) t_{b}^{3} u_{3}+\left(1-\alpha+\alpha t_{a}\right) t_{b}^{4} u \\
Y_{a}=\left(6-\beta t_{a}\right)\left(1-t_{a}\right)^{2} t_{b}^{2} v_{2}+\left[4+\alpha t_{a}+\beta\left(1-t_{a}\right)\right]\left(1-t_{a}\right) t_{b}^{3} v_{3}+\left(1-\alpha+\alpha t_{a}\right) t_{b}^{4} v \\
X_{b}=\left(4+\lambda-\lambda t_{b}\right)\left(1-t_{b}\right)^{3} t_{b} u_{1}+\left(6-\beta t_{b}\right)\left(1-t_{b}\right)^{2} t_{b}^{2} u_{2}+\left[4+\alpha t_{b}+\beta\left(1-t_{b}\right)\right] \\
\left(1-t_{b}\right) t_{b}^{3} u_{3}+\left(1-\alpha+\alpha t_{b}\right) t_{b}^{4} u \\
Y_{b}=\left(6-\beta t_{b}\right)\left(1-t_{b}\right)^{2} t_{b}^{2} v_{2}+\left[4+\alpha t_{b}+\beta\left(1-t_{b}\right)\right]\left(1-t_{b}\right) t_{b}^{3} v_{3}+\left(1-\alpha+\alpha t_{b}\right) t_{b}^{4} v
\end{array}\right.
$$



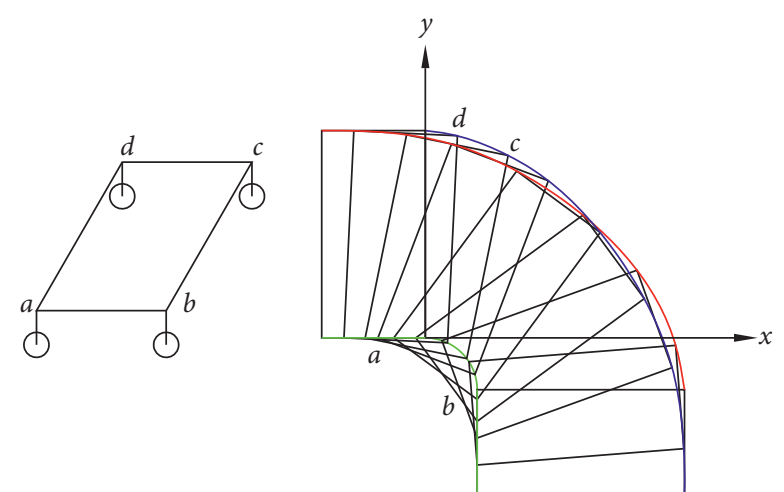

FIGURE 6: Turning of single-wheel crane.

(3) Wheels are not completely out of the curve: at this time, the coordinates of the front and rear wheels inside the crane are

$$
\left\{\begin{array}{l}
X_{a}=\left(4+\lambda-\lambda t_{a}\right)\left(1-t_{a}\right)^{3} t_{a} u_{1}+\left(6-\beta t_{a}\right)\left(1-t_{a}\right)^{2} t_{a}^{2} u_{2}+\left[4+\alpha t_{a}+\beta\left(1-t_{a}\right)\right] \\
\left(1-t_{a}\right) t_{a}^{3} u_{3}+\left(1-\alpha+\alpha t_{a}\right) t_{b}^{4} u \\
Y_{a}=\left(6-\beta t_{a}\right)\left(1-t_{a}\right)^{2} t_{a}^{2} v_{2}+\left[4+\alpha t_{a}+\beta\left(1-t_{a}\right)\right]\left(1-t_{a}\right) t_{a}^{3} v_{3}+\left(1-\alpha+\alpha t_{a}\right) t_{b}^{4} v \\
X_{b}=u+\left(v-Y_{b}\right) \cot \alpha
\end{array}\right.
$$

According to the simplified model of the running mechanism of the cart, the geometric position relationship between the support centers $a, b, c$, and $d$ can be found, and the expressions established are as follows:

$$
\begin{gathered}
\left(X_{a}-X_{b}\right)^{2}+\left(Y_{a}-Y_{b}\right)^{2}=B^{2}, \\
\left\{\begin{array}{l}
X_{c}=\frac{S}{B}\left(Y_{a}-Y_{b}\right)+X_{b}, \\
Y_{c}=\frac{S}{B}\left(X_{b}-X_{a}\right)+Y_{b},
\end{array}\right. \\
\left\{\begin{array}{l}
X_{d}=\frac{S}{B}\left(Y_{a}-Y_{b}\right)+X_{a}, \\
Y_{d}=\frac{S}{B}\left(X_{b}-X_{a}\right)+Y_{a},
\end{array}\right.
\end{gathered}
$$

where $t_{a}$ and $t_{b}$ are, respectively, the parameters of Bezier curve equation at the center of the support $a$ and $b$. Positioned at the central point of the rear wheel named as $a$, the position coordinates of the support center $b$ at different times can be obtained, respectively, by combining equations (4)-(6) with (7). Then, the position coordinates of the support center $c$ and $d$ can be obtained by equations (8) and (9).

After achieving the position coordinates of the outer front fulcrum $c$, establish the local coordinate system shown in Figure 7.
Among them, point $a$ is the origin of the local coordinate system, the angle between the $x$ ' axis of the local coordinate system and the $x$ axis of the generalized coordinate system is $\beta$, and the positive direction is counterclockwise. The transformational relations between the matrices of the trajectory coordinate of the support center $c$ in the generalized coordinate system named as $X_{c}, Y_{c}$ and those in the local coordinate system named as $X_{c}^{\prime}, X_{c}^{\prime}$ are as follows:

$$
\left\{\begin{array}{l}
X_{c}^{\prime}=\left(X_{c}-X_{a}\right) \cos \beta+\left(Y_{c}-Y_{a}\right) \sin \beta, \\
Y_{c}^{\prime}=\left(Y_{c}-Y_{a}\right) \cos \beta-\left(X_{c}-X_{a}\right) \sin \beta .
\end{array}\right.
$$

The relative coordinate matrices of the support center $c$ in the local coordinate system can be obtained through equation (10), and then the longitudinal coordinate matrix of the intersection point of the $c$-trajectory line and the vertical axis $y^{\prime}$ of the local coordinate system can be obtained by the Hermite interpolation method. Then, take the absolute value of the result of subtracting the gauge distance $S$ from the value of longitudinal coordinate matrix, the deviation matrix named as D of the outer front, and rear points at the origin of the local coordinate can be obtained by taking half of the absolute value. With the movement of the running mechanism of the cart, deviation matrix $D$ exhibits a maximum deviation value named as $M$ which affects the orbital transitivity. The movement of control points $p_{1}, p_{2}, p_{3}$ and the change of three shape parameters generate a set of inner rail curve which will produce 


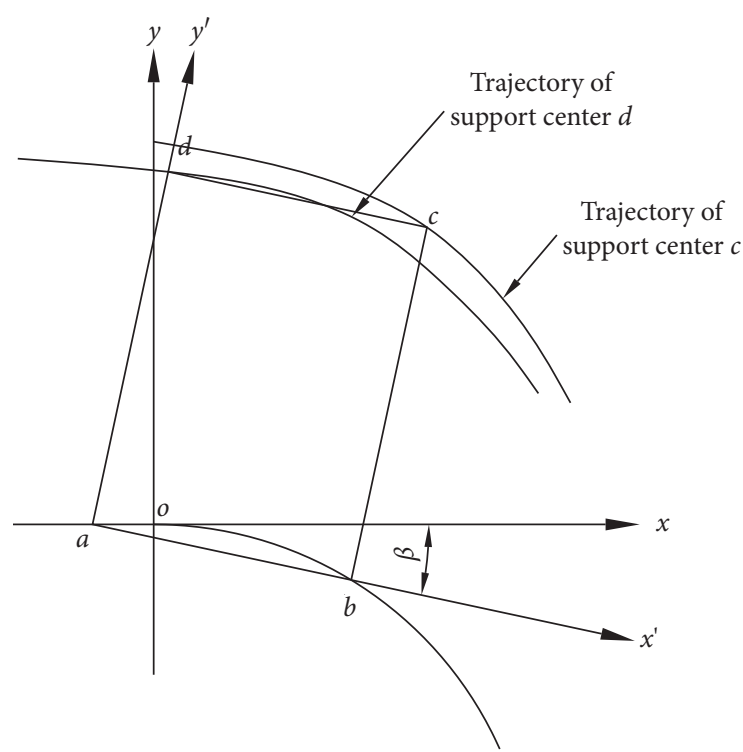

Figure 7: Local coordinate system.

different $M$. Finally, the curve corresponding to the minimum $M$ is selected as the optimal inner rail.

3.2. Multiwheel Case. There are many kinds of common multiwheel cranes, besides four-wheel, six-wheel, and eightwheel cranes. During the turning of the crane, the wheels on first-level balanced beam can only be collinear. Therefore, only the central coordinates of the collinear wheels (the position coordinates of rotating center of the collinear wheels) are calculated when establishing the mathematical models.

In the case of two-wheel crane turning, there are only two wheels fixed on each support, so it only needs to calculate the position coordinates of the each support center. Therefore, the mathematical model of the two-wheel turning is the same as that of the single-wheel case.

In the case of four-wheel crane turning, here are two cases according to whether the first-level balanced beam can rotate around the vertical shaft:

(1) Four wheels are collinear: since the four wheels can only rotate around the center of the vertical shaft on the second-level balanced beam but cannot rotate relatively, the mathematical modeling is handled in the same way as that of two-wheel turning.

(2) Two wheels are collinear: similar to the two-wheel case, it only needs to calculate the coordinates of the center position of the collinear wheels (the position coordinates of the endpoints of second-level balanced beam), and the center of the revolving shafts connected to the second-level balanced beam on the same track is at the center line of the track.

Since the constraint equations generated by whether the center of each revolving shaft enters the curve are different, a segmentation calculation process is required. Segmentation process is similar to singlewheel case, which is divided into seven situations:

(1) $b 2$ has entered the curve until $b 1$ just enters the curve.

(2) $b 1$ has entered the curve until $a 2$ just enters the curve.

(3) $a 2$ has entered the curve until $a 1$ just enters the curve.

(4) Crane completely has entered the curve until $b 2$ just leaves the curve.

(5) $b 2$ has left the curve until $b 1$ just leaves the curve.

(6) $b 1$ has left the curve until $a 2$ just leaves the curve.

(7) $a 2$ has left the curve until $a 1$ just leaves the curve.

The third case (as shown in Figure 8) is selected to be described in detail, while the other cases are similar and will not be described again.

When the centers of the revolving shafts named as $a_{2}$, $b_{1}$, and $b_{2}$ are all on the curved track, the constraints of their horizontal and vertical coordinates named as $X_{a 2} Y_{a 2}, X_{b 1} Y_{b 1}$, and $X_{b 2} Y_{b 2}$ all satisfy equation (3), and $t_{a 2}, t_{b 1}$, and $t_{b 2}$ correspond to the three parameters of the Bezier curve's equation at the points $a_{2}, b_{1}$, and $b_{2}$, respectively.

According to the geometric relationship of the fourwheel crane's cart mechanism, the coordinate equations of the inner revolving centers are established:

$$
\left\{\begin{array}{l}
X_{a_{2}}=X_{a_{1}}+K_{2} \cos \theta \\
Y_{a_{2}}=Y_{a_{1}}+K_{2} \sin \theta \\
X_{b_{1}}=X_{a_{1}}+\frac{K_{2}}{2} \cos \theta+B \cos \beta-\frac{K_{2}}{2} \cos \gamma, \\
Y_{b_{1}}=Y_{a_{1}}+\frac{K_{2}}{2} \sin \theta+B \sin \beta-\frac{K_{2}}{2} \sin \gamma, \\
X_{b_{2}}=X_{a_{1}}+\frac{K_{2}}{2} \cos \theta+B \cos \beta+\frac{K_{2}}{2} \cos \gamma, \\
Y_{b_{2}}=Y_{a_{1}}+\frac{K_{2}}{2} \sin \theta+B \sin \beta+\frac{K_{2}}{2} \sin \gamma
\end{array}\right.
$$

where $\beta, \gamma$, and $\theta$ are the angles between the inner lower balanced beam named as $a b$, the second-level balanced beams named as $b_{1} b_{2}, a_{1} a_{2}$, and the positive direction of $x$ axis, respectively, and the positive direction is counterclockwise.

Positioned at the center of the revolving shaft named as $a_{1}$, equation (11) is connected to the constrained equations of the horizontal and vertical coordinate of $a_{2}, b_{1}$, and $b_{2}$ which contain six unknown parameters $\beta$, 


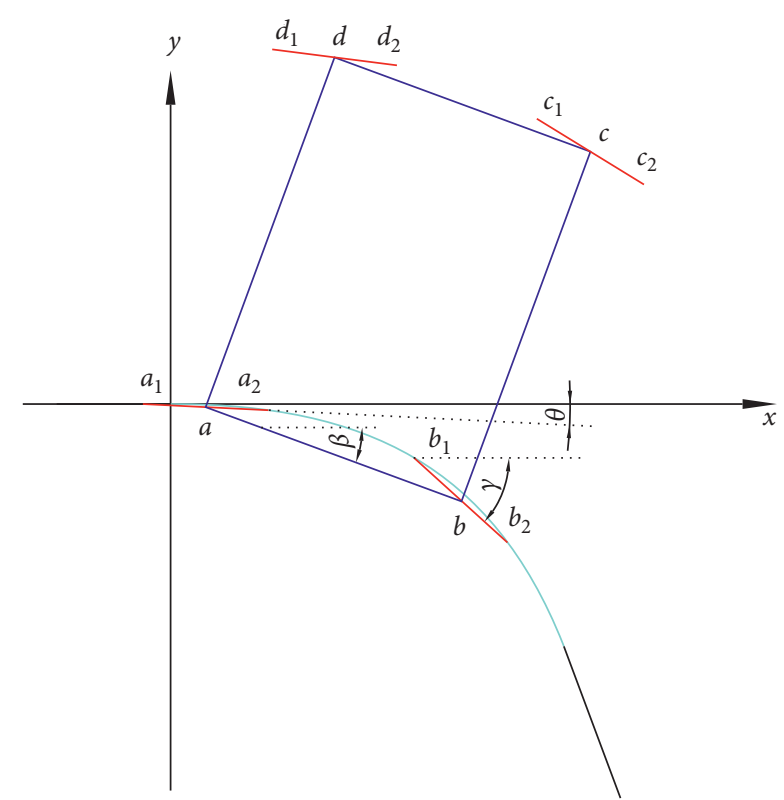

Figure 8: Turning of four-wheel crane when the two wheels are collinear.

$\gamma, \theta, t_{a 2}, t_{b 1}$, and $t_{b 2}$, and the number of the unknown parameters is the same as that of the independent equations, so the coordinates of the central trajectory of the inner support can be obtained. The calculation of the coordinates of the point $c$ and $d$ and the deviation of the outer front and rear points are consistent with the single-wheel case.

In the same way, the theoretical methods and situational discussion of the turning of the six-wheel and eight-wheel carts are similar to those of the singlewheel and four-wheel cases, and it will not be repeated here.

\section{Optimal Calculation of Inner Rail Based on Global Optimization Algorithm}

The noncircular inner rail formed by the quasiquartic Bezier curve with three shape parameters is continuously optimized by the position of five control points and the change of parameters. The goal of optimization is to minimize the value of $M$. At this point, the position coordinates of the control points and the value of the three parameters can form the optimal inner rail. However, in most practical engineering applications, the noncircular inner rails are usually designed symmetrically according to the natural conditions of the port shore, and its specific performance in Figure 5 is as follows: due to the limitations of the natural conditions of each port site, two straight lines derived from the straight tracks intersect at the fixed point $A$ and the complementary angle $a$ of the angle between two lines is known. Then, point $A$ is taken as the center of a circle. According to the actual area of the installation site, the land occupation of the noncircular inner rail, the size of the turning radius, and the engineering requirements, a reasonable radius is chosen to form a circle, and the intersection of the circle and the straight tracks I and II, respectively, fixes the positions of the control points $p_{0}$ and $p_{4}$, so the shadow area is an isosceles triangle named as $\triangle P_{0} \mathrm{AP}_{4}$. So far, the change of the position of the control points $p_{1}, p_{2}$, and $p_{3}$ and the parameters $\lambda, \alpha$, and $\beta$ determine the shape of the inner rail, thus affecting the value of the optimization target $M$.

Generally speaking, the optimization problem of crane turning on the noncircular inner rail can be expressed as follows:

$$
\text { Global min } M\left(P_{1}\left(u_{1}, v_{1}\right), P_{2}\left(u_{2}, v_{2}\right), P_{3}\left(u_{3}, v_{3}\right), \lambda, \alpha, \beta\right) \text {. }
$$

It can be seen from Figure 5 that the control points $p_{1}$ and $p_{3}$ move randomly on the line segments $\mathrm{AP}_{0}$ and $\mathrm{AP}_{4}$. In view of the symmetry design of the inner rail, the optimal trajectory of the point $p_{2}$ can be determined on the line connecting the point $A$ and the midpoint of the $p_{0} p_{4}$. Therefore, the following relationships can be established:

$$
\left\{\begin{array}{l}
z=u+v \cot \alpha \\
v_{2}=\frac{v\left(u_{2}-z\right)}{(u-2 z)} \\
u_{3}=z-v_{3} \cot \alpha
\end{array}\right.
$$

Then, the global optimization problem of the inner rail can be simplified as follows:

$$
\begin{array}{cl}
\text { Global } & M\left(u_{1}, u_{2}, v_{3}, \lambda, \alpha, \beta\right), \\
\text { s.t. } & \left\{\begin{array}{l}
0 \leq u_{1} \leq z ; \frac{u}{2} \leq u_{2} \leq z ; \nu \leq v_{3} \leq 0, \\
-4 \leq \lambda \leq 1 ;-4 \leq \beta \leq 6 ;-4 \leq \alpha \leq 1 .
\end{array}\right.
\end{array}
$$

In summary, the optimization problem of the inner rail contains multiple variables, with nonlinear, nonconvex features and multiple constraints. The objective function is discontinuous, nondifferentiable, and highly nonlinear. In addition, it has no explicit analytical expression, so the classic local optimization method is no longer applicable. Although the local optimization algorithm is much more mature than the global optimization algorithm and is easy to be implemented in general, its results completely depend on the selection of neighborhood function and initial points. It is easy to fall into the local minimum during the search process, so it cannot be realized in most cases of global optimization.

At present, the global optimization algorithms developed at home and abroad generally fall into two types: deterministic and random. The former includes the branch and bound method, integration method on level set, falling trajectory method, tunnel method, sequence method, and filling function method, while the latter includes the uniform distribution search method, multistart algorithm, clustering algorithm, simulated annealing, tabu search, genetic 
algorithm, neural network, ant colony algorithm, particle swarm algorithm, and so on. GlobalSearch algorithm in the toolbox of Matlab, which belongs to the multistart heuristic algorithm, is chosen as the method this time. Its basic principle is that first, the global search solver generates several initial testing points based on distributed search; second, these initial points are used to call the local solver named as fmincon to continually discriminate and search the extreme points in their respective basins through the SQP (sequence quadratic programming) algorithm and the starting points are deleted with little hope of success; finally, the evaluation function is used to compare the extreme points of each basin to get the global optimal value. It has been proved by many experiments that the GlobalSearch algorithm can not only obtain more accurate, stable, and reliable value of $M$ but also reduce the time of program operation compared with other global optimization algorithms.

\section{Case Calculation and Analysis}

At present, there are no international standards for the basic parameters of cranes. In practical engineering, countries, regions, and even wharfs will design and determine themselves according to different requirements. However, some basic parameters of quayside container cranes have been formed in various regions of the world, such as gauge distance, base distance, and so on. Combining with the actual project, according to the current existing parameters of quayside container cranes, the following data of quayside container cranes and rails are selected: track angle $a=70^{\circ} \sim 90^{\circ}$; gauge distance $S=20 \mathrm{~m} \sim 35 \mathrm{~m}$; base distance $B=10 \mathrm{~m} \sim 15 \mathrm{~m}$; wheel distance $K_{0}=1 \mathrm{~m}$; and radius of arc inner rail $R=120 \mathrm{~m} \sim 150 \mathrm{~m}$.

5.1. Comparative Analysis of Rail Optimization in SingleWheel and Multiwheel Cases. According to the model of crane turning established above, by selecting the track angle $a=90^{\circ}$, the gauge distance $S=30 \mathrm{~m}$, the base distance $B=15 \mathrm{~m}$, the track $K_{0}=1 \mathrm{~m}$, and the radius of arc inner rail $R=120 \mathrm{~m}$ and calculating by global optimization algorithm, the curve parameters of inner rail and corresponding deviations of different cranes shown in Table 1 are obtained.

As shown in Table 1, it is not difficult to see that regardless of the type of crane, the parameters and deviations of the final optimization returned are very close. In addition, although the points $p_{1}$ and $p_{3}$ move randomly on $\mathrm{AP}_{0}$ and $\mathrm{AP}_{4}$, the optimal position of the two points is basically symmetrical about the median line, which also confirms that the optimal trajectory of point $p_{2}$ is on the line connecting point $A$ and the midpoint of $p_{0} p_{4}$. At the same time, it can be seen from Figure 9 that the inner and outer rail trajectories and deviation maps in single-wheel and multiwheel cases are almost coincident. In order to facilitate the comparison of the circular rail, quartic Bezier curve track, and quasiquartic Bezier curve track with three shape parameters and to analyze and discuss the effect of different parameters on the deviation of noncircular inner rail, the single-wheel crane is selected as the main research object.

\subsection{Comparative Analysis of the Deviations between Circular} and Noncircular Rails. Under the same turning parameters, the comparison of the inner and outer rail trajectories and the deviations of outer front and rear points between the circular rail, quartic Bezier curve track, and quasiquartic Bezier curve track is shown in Figure 10.

When the crane runs on the circular rail, the maximum deviation is $22.49 \mathrm{~mm}$ at the start of the curve, and the deviation is zero after entering the curve completely, which means the front and rear trajectories coincide and no deviation problem occurs, and a larger deviation occurs again when the crane just leaves the curve. When the Bezier curve is used as the inner rail, the maximum deviation will not exceed $4.7 \mathrm{~mm}$, and the deviation of trajectory is very small and smooth during the turning process, especially avoiding the sharp increase of the deviation when the cart enters and leaves the curve, thus greatly reducing the probability of jamming and gnawing when the cart just enters and leaves the curve and highlighting the superiority of the Bezier curve as the inner rail.

After further observation of Figure 10, it is found that the inner and outer trajectories are almost coincident when using quartic Bezier curve and quasiquartic Bezier curve with three shape parameters as inner rails, and their shape is only slightly different in the local position, but there are obvious differences in the deviations. When the quasiquartic Bezier curve is chosen as the inner rail, the maximum deviation is $4.3645 \mathrm{~mm}$, which is smaller than the maximum deviation $(4.6819 \mathrm{~mm})$ when the quartic Bezier curve is used as the inner rail, and the overall trend of the deviation is more stable, which guarantees the traceability of the front and rear wheels on the outside. Therefore, it will achieve better results in avoiding and eliminating the hidden dangers that wheels gnaw and jam rails.

\subsection{Influence of the Parameters of the Crane Cart on the} Deviation. It is not difficult to find out from the model of crane turning that the parameters affecting the deviation are the base distance $B$, the gauge distance $S$, the radius $R$ of arc inner rail, and the angle $a$ between two straight rails. By analyzing the influence of these parameters on the turning of the crane, the variation law of the deviation can be further understood.

Figure 11 shows the variation trend of the deviation of the inner rail based on quasiquartic Bezier curve under different parameters. It can be seen that with the increase of the base distance $B$ and the gauge distance $S$, the deviation also shows an upward trend. However, the radius $R$ of arc inner rail and the angle $a$ between two straight rails are inversely proportional to the deviation, that is, the increase of $R$ and $a$ will reduce the value of the deviation. Nevertheless, no matter what kind of parameters, the maximum deviation $M$ of the noncircular rail based on Bezier curve is 
TABle 1: Optimization parameters and deviations of different cranes' inner rail.

\begin{tabular}{|c|c|c|c|c|c|c|c|}
\hline Type of crane & $u_{1}(\mathrm{~mm})$ & $u_{2}(\mathrm{~mm})$ & $V_{3}(\mathrm{~mm})$ & $\lambda$ & $\alpha$ & $\beta$ & $M(\mathrm{~mm})$ \\
\hline Single-wheel & 38910 & 116490 & -81083 & 0.5712 & 0.4741 & 0.4408 & 4.3645 \\
\hline Four-wheel & 39258 & 116170 & -80739 & 0.5939 & 0.4851 & 0.4756 & 4.3373 \\
\hline
\end{tabular}

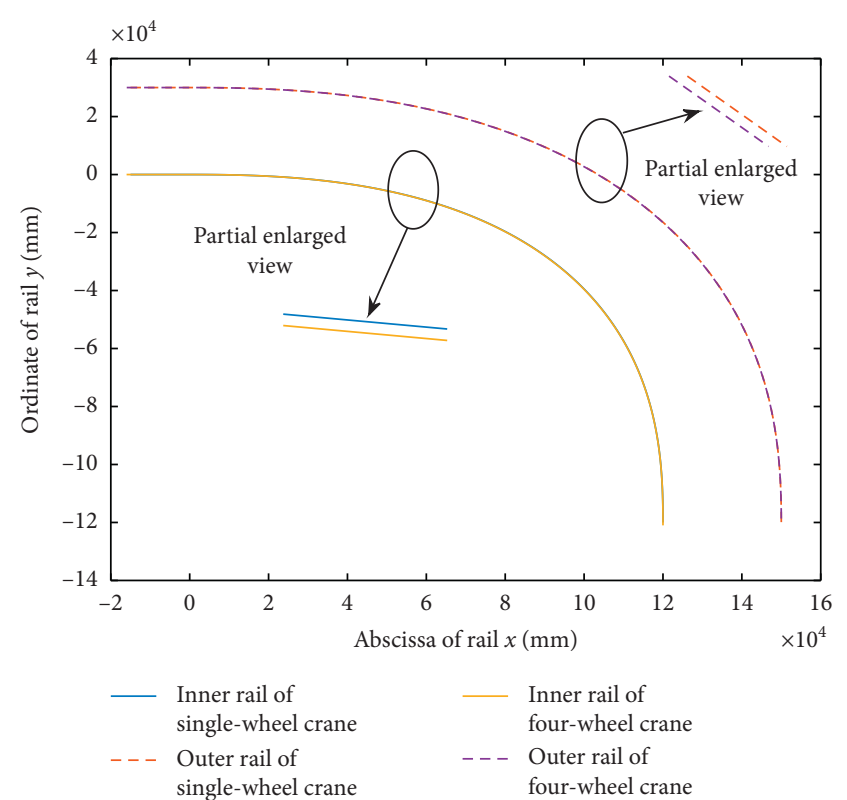

(a)

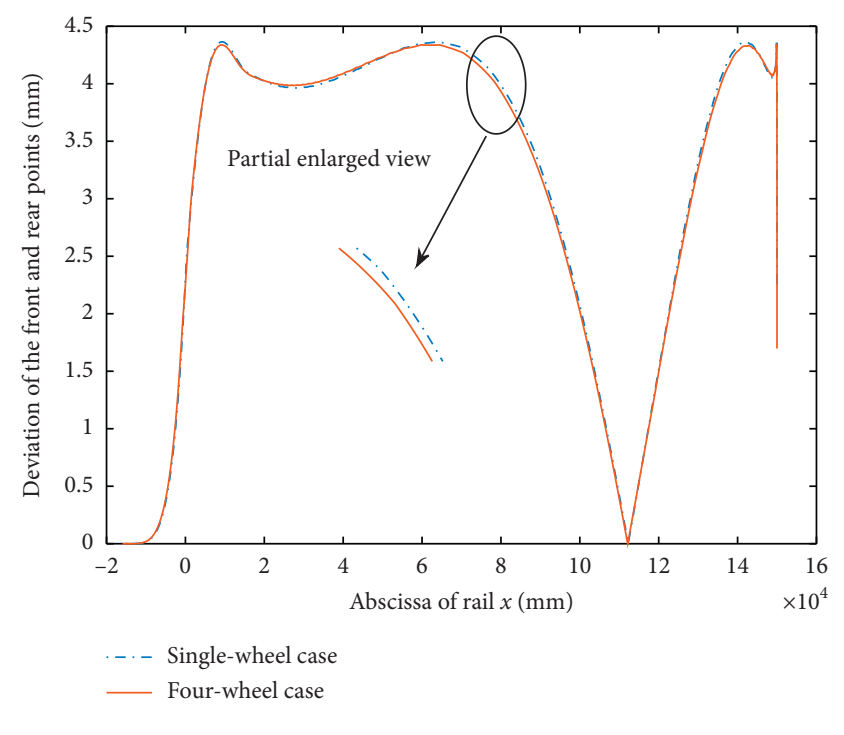

(b)

FIGURE 9: Optimized inner and outer rail trajectories and deviation maps in single-wheel and multiwheel cases.

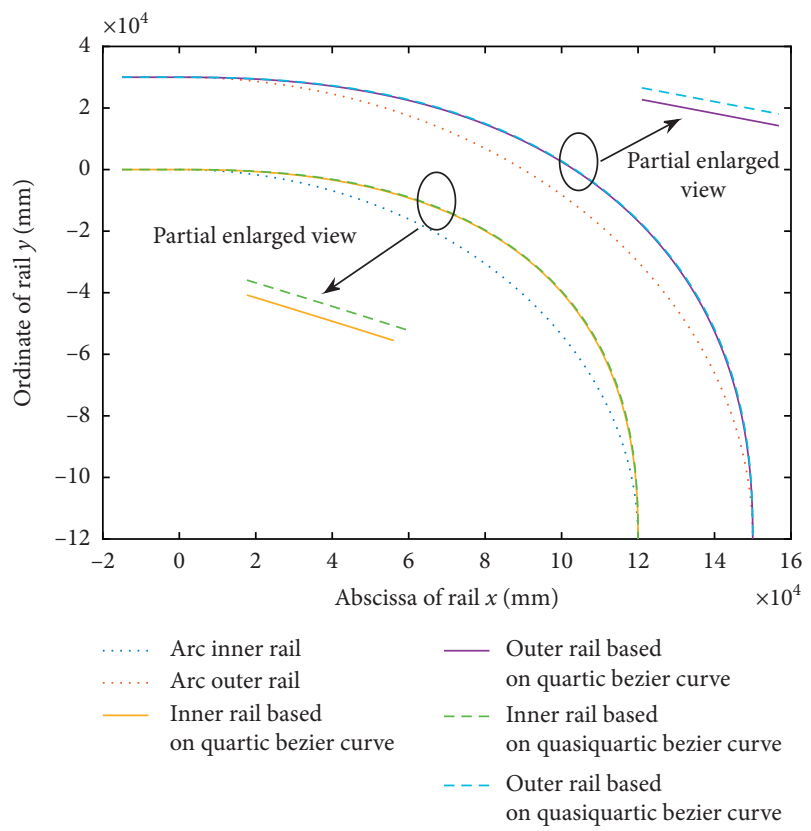

(a)

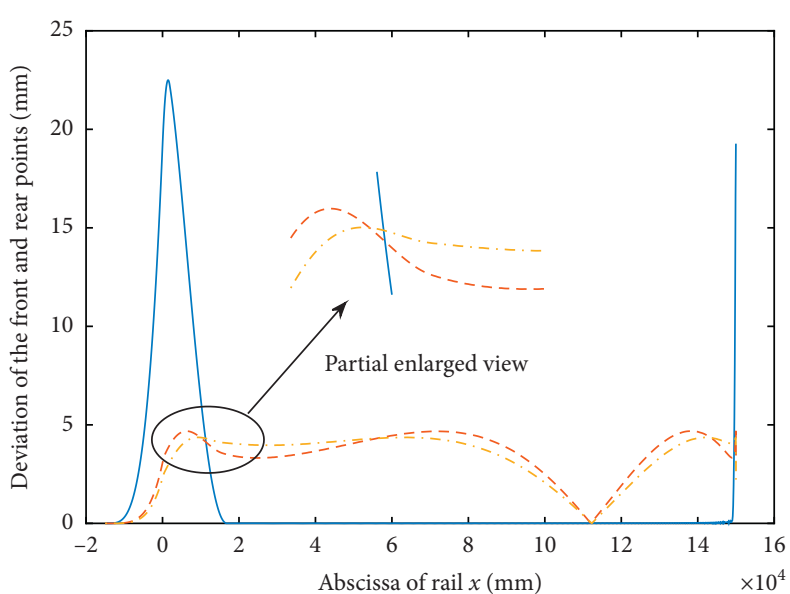

- Arc inner rail

- - - Inner rail based on quartic bezier curve

Inner rail based on quasiquartic bezier curve

(b)

FIGURE 10: Comparison of trajectories and the deviations of front and rear points. (a) Comparison of trajectories. (b) Comparison of the deviations of front and rear points. 

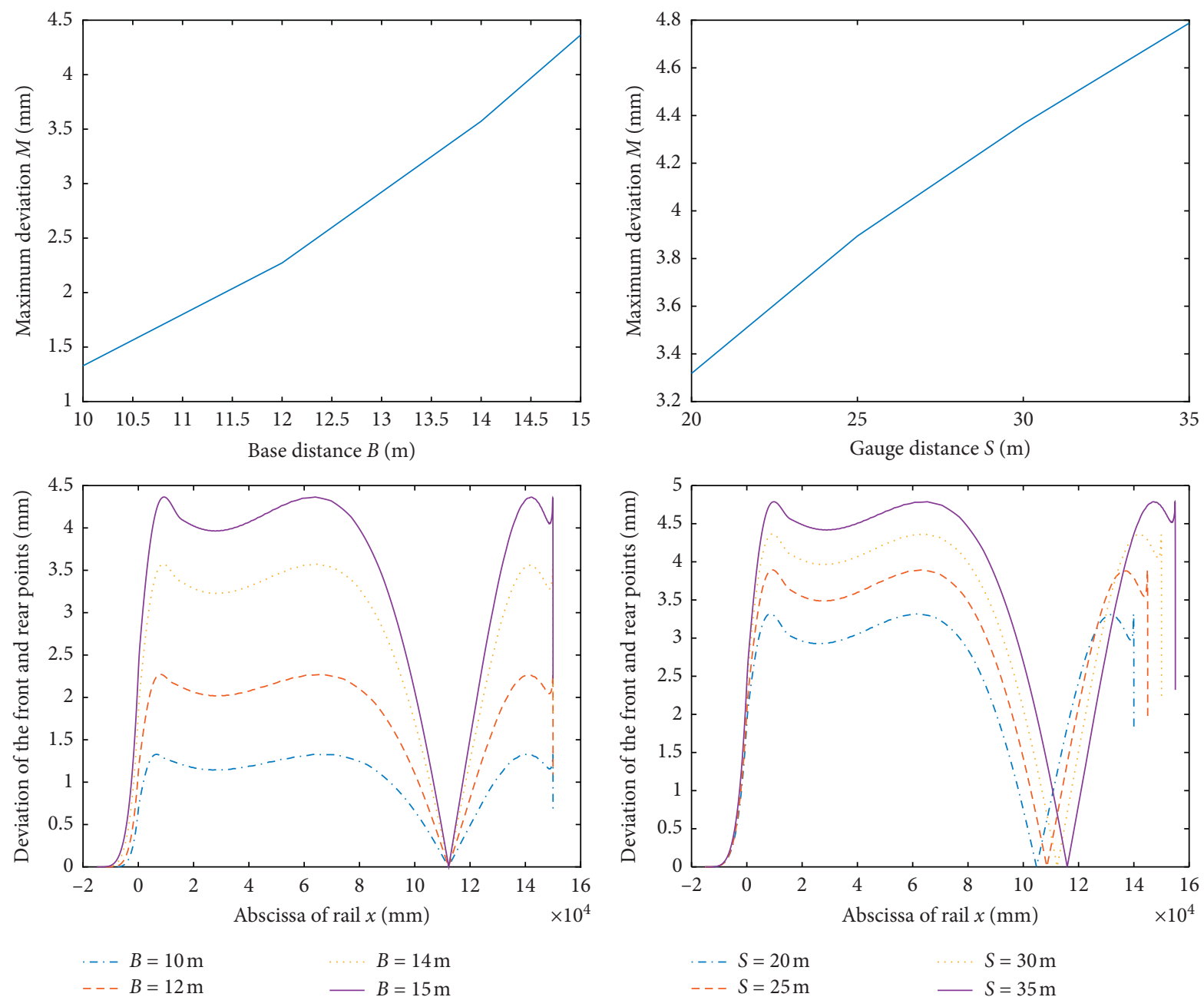

$\begin{aligned} \cdots & =20 \mathrm{~m} \\ ---S & =25 \mathrm{~m}\end{aligned}$

$S=30 \mathrm{~m}$

(a)

(b)

Figure 11: Continued. 

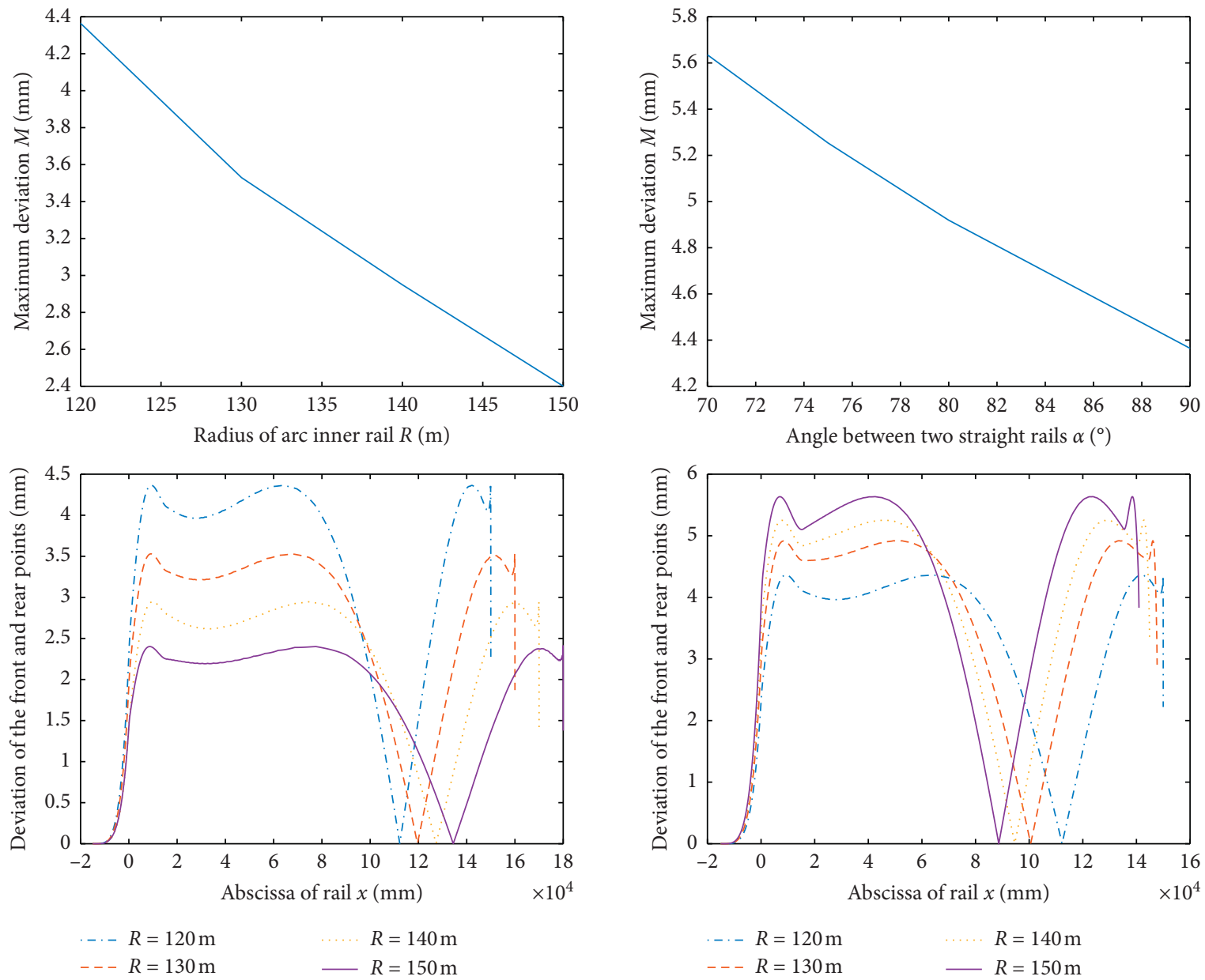

(c)

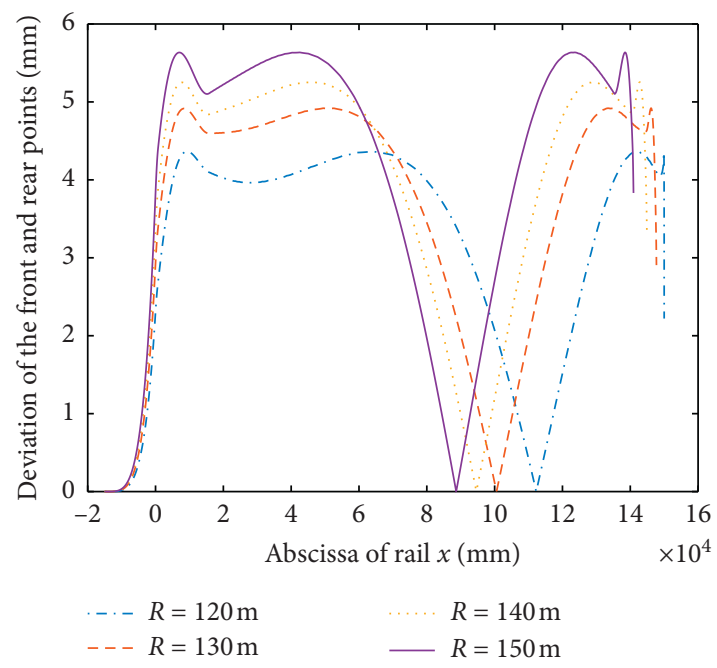

(d)

FigURE 11: Diagrams of the deviation under different parameters. (a) Variation of the deviation at different base distances (B). (b) Variation of the deviation at different gauge distances (S). (c) Variation of the deviation at different radii (R) of arc inner rails. (d) Variation of the deviation at different angles (a) between two straight rails.

much smaller than that of the circular rail, which has great advantages.

5.4. Dynamic Simulation Experiment Based on ADAMS. Take the traveling mechanism of a single-wheel crane as an example. Because the metal structure of the crane has no effect on the simulation, it is directly simplified into a frame form. According to the parameters determined above and the data obtained after optimization, establish the corresponding 3D model in SolidWorks software. The wheels are cylindrical with double rims. The diameter of wheel tread is $800 \mathrm{~mm}$, the diameter of outer edge of wheel is $850 \mathrm{~mm}$, the width of rail top surface is $100 \mathrm{~mm}$, and the width of wheel tread is $125 \mathrm{~mm}$. Import the 3D model into ADAMS in $\mathrm{x} \_\mathrm{t}$ format and add material properties to each part and corresponding constraints according to Table 2, and the final model is shown in Figure 12.

Add a constant speed $(750 \mathrm{~mm} / \mathrm{s})$ to the frame in the $x$ axis direction. After setting the end time and steps of simulation, start the simulation and view the results through the postprocessor interface.

As shown in Figure 13, the eight wheels are kept in contact with two rails during the turning process of the crane, and no rail jamming and gnawing occurred. The contact force fluctuates up and down by $375 \mathrm{KN}$. Therefore, the traveling mechanism of the crane cart can run safely and stably on the optimized rails.

After further observation of Figure 14, it is found that the left and right parts of wheels do not contact with the sides of 
TABle 2: Types of constraints between parts.

\begin{tabular}{lccc}
\hline Part name & Part name & Constraint type & Number of constraints \\
\hline Inner rail & Ground & Fixed joint & 1 \\
Outer rail & Ground & Fixed joint & 1 \\
Left part of wheel & Middle part of wheel & Fixed joint & 4 \\
Right part of wheel & Middle part of wheel & Fixed joint & 4 \\
Middle part of wheel & Wheel shaft & Fixed joint & 4 \\
Wheel shaft & Balanced beam & Revolute joint & 4 \\
Balanced beam & Frame & Revolute joint & 4 \\
\hline
\end{tabular}

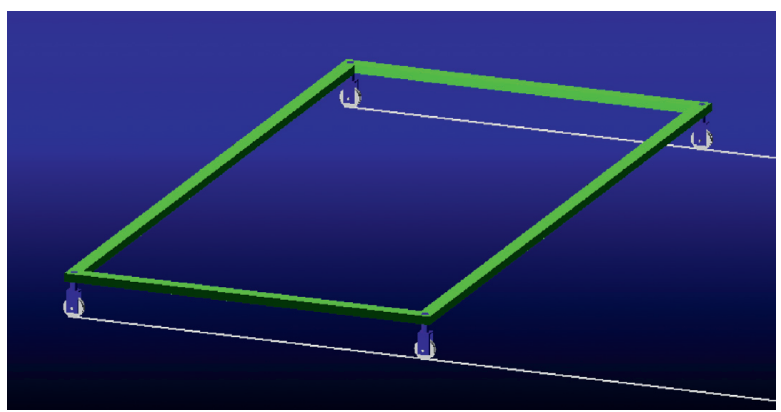

(a)

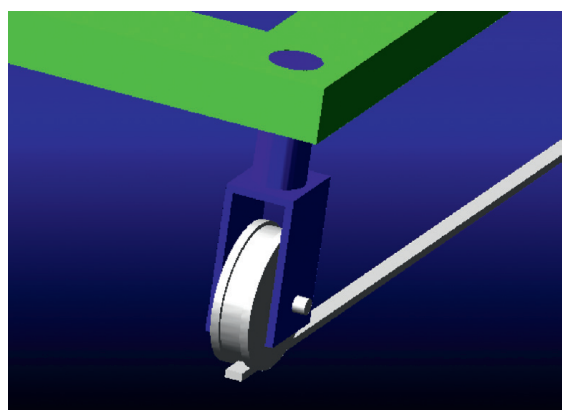

(b)

Figure 12: Simplified simulation model of traveling mechanism of single-wheeled cart. (a) Turning system structure. (b) Single-wheel structure.

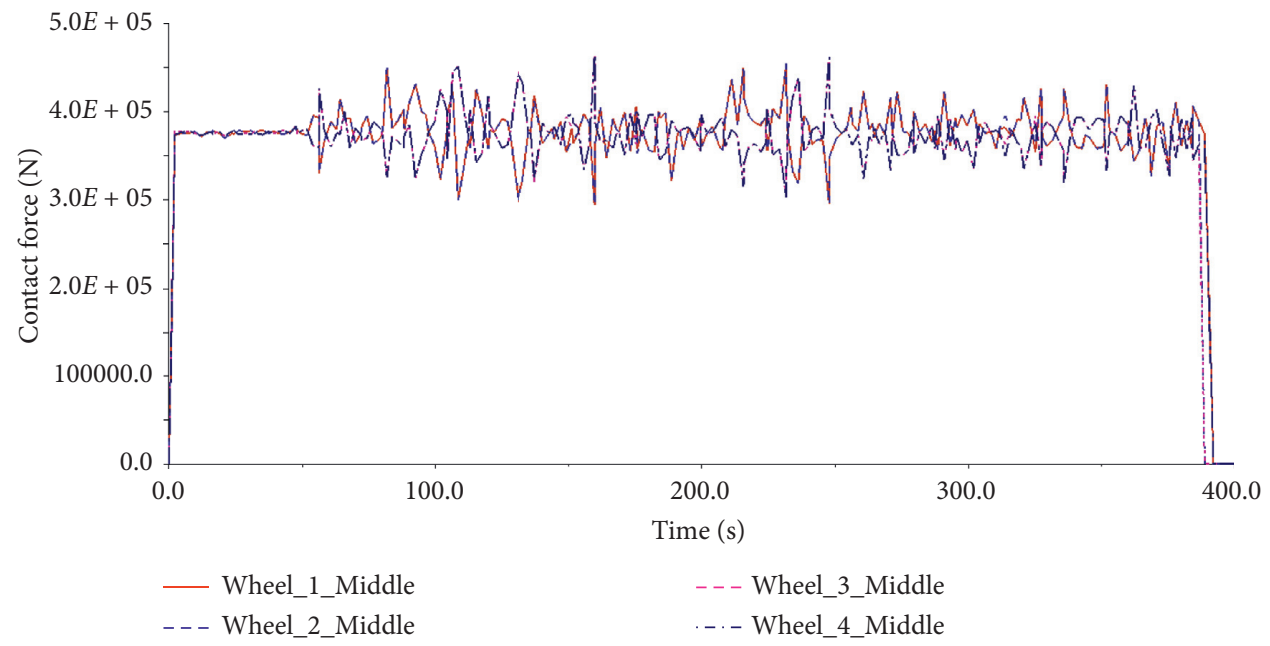

Figure 13: Contact force between middle part of wheel and top surface of rail. 


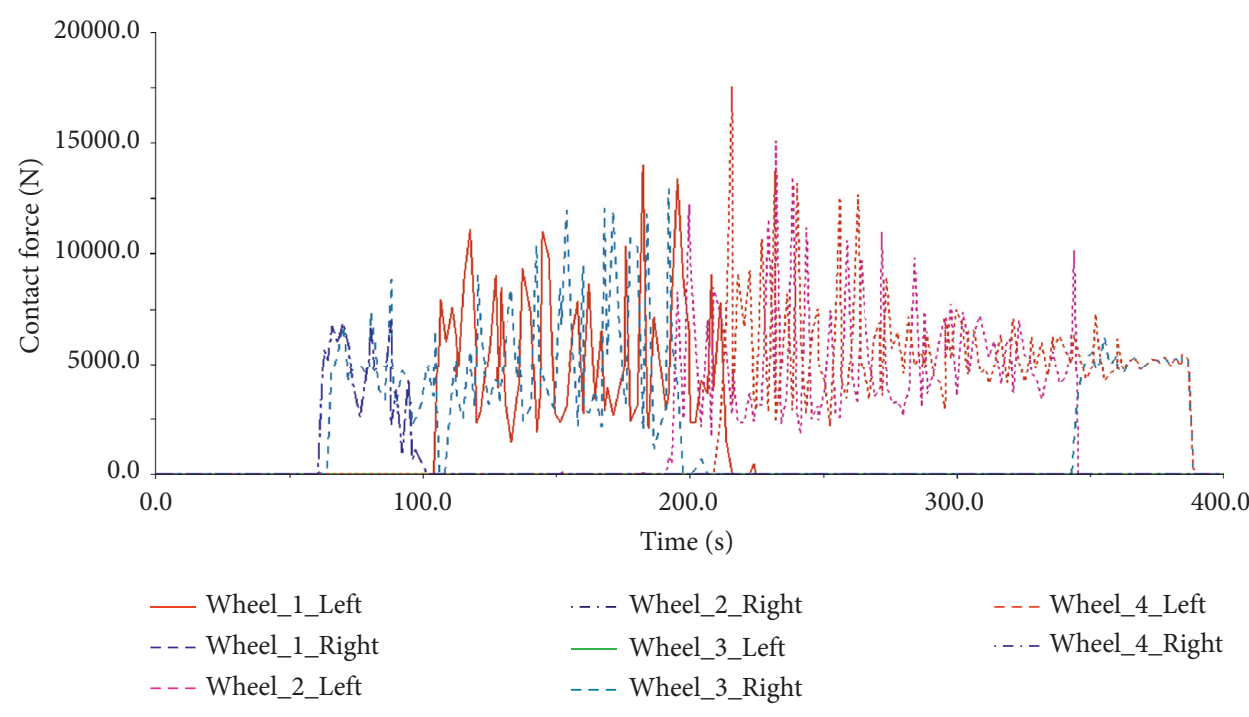

FIGURE 14: Contact force between wheel side and rail side.

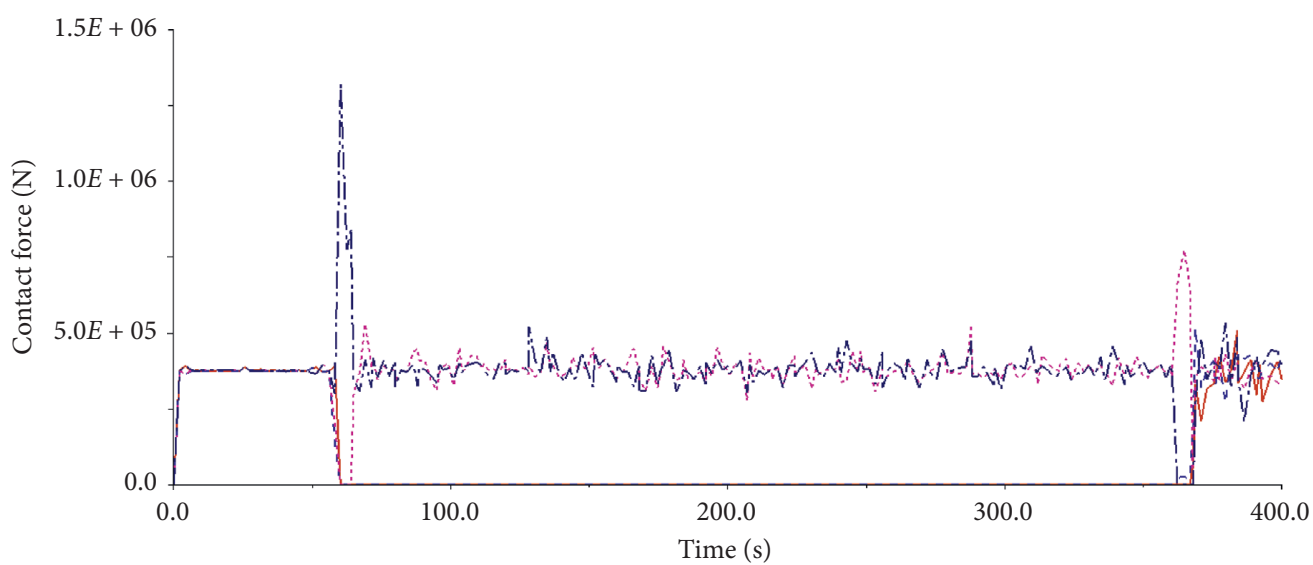

— Wheel_1_Middle

- - - Wheel_2_Middle

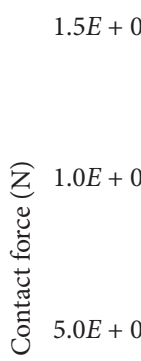

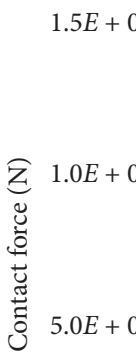

0.0

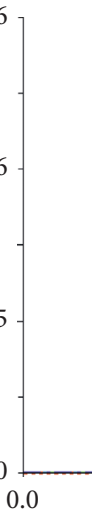

— Wheel_1_Left

- - - Wheel_1_Right

- - - Wheel_2_Left
- - - Wheel_3_Middle

-..-Wheel_4_Middle

(a) 
the crane cart runs on the curved rail, different wheels have contact forces with the sides of rails at different times. Among them, the left part of wheel 4 generates a maximum contact force about $17574 \mathrm{~N}$ with the side of rail at 215 seconds.

Figure 15 shows the contact force between wheels and rails when crane turns on the arc rails. The left part of wheel 2 and wheel 3, respectively, generates about $1481.7 \mathrm{KN}$ and 1286.2 KN contact force when crane just enters arc rails, and the left part of wheel 1 generates about $882.1 \mathrm{KN}$ contact force when crane leaves arc rails. Therefore, compared with crane running on the optimized track, it is not difficult to find that wheels have a greater contact force when entering and exiting arc rails, which will cause wheels to jam and gnaw rails. In this case, wheel 2 and wheel 3 are more likely to jam and gnaw rails than other wheels, especially wheel 2.

\section{Conclusion}

(1) In the rail optimization of noncircular curve of crane turning, the Bezier curve is introduced in this paper. Taking the minimum deviation of the outer front and back points as the optimized target, the global optimization algorithm is used to search for the best inner rail. Finally, the trajectory of outer rail is fitted by interpolation method. By comparing the quartic Bezier curve as the inner rail with the traditional circular rail, the results show that the deviation of the circular inner rail is several times larger than that of the inner rail based on quartic Bezier curve when the crane just enters and leaves the curve, which is also an important reason for crane jamming and gnawing rails. This highlights the advantage of the Bezier curve as the inner rail of crane turning.

(2) In order to further reduce the deviation, a quasiquartic Bezier curve with three shape parameters is introduced in this paper. Similarly, the algorithm is used to find the optimal position of the control points and the optimal value of three parameters which form the best inner rail. The results show that the three shape parameters have different effects on the inner rail, and the inner rail can be fine-tuned by changing the value of the parameters. Therefore, compared with the quartic Bezier curve, using the quasiquartic Bezier curve with three shape parameters as the inner rail not only further reduces the maximum deviation but also makes the deviation of the front and rear points of the outer rail tend to be stable, which guarantees the traceability of the outer front and rear wheels in the course of traveling and reduces the probability of wheels jamming and gnawing rails.

(3) In addition, it is also analyzed that the deviation will increase with the increase of the base distance $B$ and the gauge distance $S$ and decrease with the increase of the radius $R$ of arc inner rail and the angle $a$ between two straight rails. Finally, the practical feasibility of the scheme is verified through dynamic simulation experiments. The deviation of the crane track has a great influence on the safety of the actual engineering operation. Taking the quasiquartic Bezier curve with three shape parameters as the inner rail can bring certain reference value to the actual production.

\section{Data Availability}

The data used to support the findings of this study are included within the article.

\section{Conflicts of Interest}

The authors declare that they have no conflicts of interest.

\section{References}

[1] S. Z. Gu, "Turning design of operating mechanism of gantry crane in hydropower station," Hoisting and Conveying Machinery, vol. 6, pp. 33-38, 1977.

[2] Q. X. Ni, "Turning problem of rail-operated crane," Hoisting and Conveying Machinery, vol. 3, pp. 66-78, 1980.

[3] G. L. Xu, "Some questions about analysis and calculation of crane's turning," Construction Machinery, vol. 5, pp. 8-12, 1995.

[4] Z. W. Zhang, H. Q. Yu, J. N. Wang, and Q. F. Bao, Crane Design Manual, China Railway Publishing House, Beijing, China, 1997.

[5] W. T. Wang, "Large-scale round-tracked crane design," Chinese Journal of Construction Machinery, vol. 6, no. 2, pp. 210-213, 2008.

[6] H. Gao, Design and Application of Transition Curve, Zhejiang University of Technology, Hangzhou, China, 2015.

[7] H. Wang, "Programming implementation of various generalized calculation models for alleviation curves," in Proceedings of the 5th China International Tunnel Engineering Symposium, pp. 996-1002, Shanghai, China, 2011.

[8] W. G. Xu, Z. H. Long, and Z. W. Yu, "Methods of coordination calculation of different spiral curves," Journal of Heilongjiang Institute of Technology, vol. 27, no. 3, pp. 20-23, 2013.

[9] E. Soderberg and M. Jordan, Transferring Container Cranes Around Corners Using Curved Rails-Design Considerations, pp. 1-10, ACSE, Reston, VA, USA, 2001.

[10] J. L. Chen, D. S. Dong, and Z. Qiao, "Non-circular crane rail theory and parametric design," Mathematical Modelling of Engineering Problems, vol. 4, no. 1, pp. 48-52, 2017.

[11] Y. C. Huang, "Application of rational Bézier curve in the path planning of robots," Smart Factory, vol. 6, pp. 53-57, 2015.

[12] C. Cheng, Y. Q. He, C. G. Bu, and J. D. Han, "Feasible trajectory generation for autonomous vehicles based on quartic Bézier Curve," Acta Automatica Sinica, vol. 41, no. 3, pp. 486-496, 2015.

[13] L. Han, H. Yashiro, H. T. N. Nejad, H. D. Quoc, and S. Mita, "Bézier curve based path planning for autonomous vehicle in urban environment," in Proceedings of the 2010 IEEE Intelligent Vehicles Symposium, pp. 1036-1042, San Diego, CA, USA, June 2010.

[14] I. Bae, J. Moon, H. Park, J. H. Kim, and S. Kim, "Path generation and tracking based on a Bézier curve for a steering 
rate controller of autonomous vehicles," in Proceedings of the 16th International IEEE Conference on Intelligent Transportation Systems (ITSC 2013), pp. 436-441, The Hague, Netherlands, October 2013.

[15] X. L. Han and S. J. Liu, "Extension of quadratic Bezier curve," Journal of Central South University (Science and Technology), vol. 34, no. 2, pp. 214-217, 2003.

[16] H. J. Hang, J. Yu, and W. G. Li, "Two parameters extension of cubic Bezier curve and its applications," Computer Engineering and Applications, vol. 46, no. 31, pp. 178-180+205, 2010.

[17] X. Q. Wu, X. L. Han, and S. M. Luo, "Two different extensions of quartic Bezier curve," Journal of Engineering Graphics, vol. 5, pp. 59-64, 2006.

[18] X. Q. Liu and G. Y. Yang, "Extension of quartic Bezier curve with two shape parameters," Journal of Graphics, vol. 34, no. 1, pp. 41-45, 2013.

[19] R. Qiu, H. J. Hang, and J. C. Pan, "Study of quasi quartic Bezier curves with three shape parameters and its application," Computer Engineering and Applications, vol. 50, no. 20, pp. 158-162, 2014.

[20] G. C. Zhang, F. Zhao, and H. Y. Xi, "Quartic Bezier curves with three shape parameters," Journal of Northwest Normal University (Nature Science), vol. 50, no. 2, pp. 26-29+34, 2014.

[21] E. T. Liu, H. M. Liu, and Z. X. Liu, "Multistart global optimization algorithm based on simplex gradient," Journal of Jilin University, vol. 54, no. 6, pp. 1282-1288, 2016.

[22] F. Y. Fu, Y. P. Tian, and S. S. Zhong, "Heuristic search algorithm for opportunistic replacement strategy of multiple life-limited parts," Journal of Aerospace Power, vol. 32, no. 8, pp. 1971-1977, 2017. 